\title{
Response times of ambulances to calls from Midwife Obstetric Units of the Peninsula Maternal and Neonatal Service (PMNS) in Cape Town
}

\author{
JK Marcus, PGDipN (Advanced Midwifery) RA, RN, RCHN, RPN \\ Division of Nursing and Midwifery, School of Health and Rehabilitation Sciences, Faculty of Health Sciences \\ University of Cape Town
}

SE Clow, MSc (Nursing), BSocSc, AUDNE, RN, RM, RCHN

Associate Professor, Division of Nursing and Midwifery, School of Health and Rehabilitation Sciences, Faculty of Health Sciences, University of Cape Town

\section{Key words}

MOU, ambulance, response time, PMNS

\section{Correspondence address}

Jason Marcus

Clinical Skills Centre

Faculty of Health Sciences

University of Cape Town

Anzio Road

Observatory

7925

Tel: (021) 4066704

Fax: (021) 4486815

Email: Jason.Marcus@uct.ac.za

\section{Abstract: Curationis 32 (1): 59-66}

Response times of ambulances to calls from Midwife Obstetric Units, although varied, are perceived as slow. Delays in transporting women experiencing complications during or after their pregnancies to higher levels of care may have negative consequences such as fetal, neonatal or maternal morbidity or death.

An exploratory descriptive study was undertaken to investigate the response times of ambulances of the Western Cape Emergency Medical Services to calls from midwife obstetric units (MOUs) in the Peninsula Maternal and Neonatal Services (PMNS) in Cape Town. Response times were calculated from data collected in specific MOUs using a specifically developed instrument. Recorded data included time of call placed requesting transfer, diagnosis or reason for transfer, priority of call and the time of arrival of ambulance to the requesting facility. Mean, median and range of response times, in minutes, to various MOUs and priorities of calls were calculated. These were then compared using the Kruskal-Wallis test. A comparison was then made between the recorded and analysed response times to national norms and recommendations for ambulance response times and maternal transfer response times respectively.

A wide range of response times was noted for the whole sample. Median response times across all priorities of calls and to all MOUs in sample fell short of national norms and recommendations. No statistical differences were noted between various priorities of calls and MOUs.

The perception of delayed response times of ambulances to MOUs in the PMNS was confirmed in this pilot study. 


\section{Introduction}

The Peninsula Maternal \& Neonatal Service (PMNS) was established in 1980 by the Department of Obstetrics and Gynaecology at the University of Cape Town with the support of the then Cape Provincial Administration (Van Coeverden De Groot, 1993: 225). The service provides "tiered, comprehensive" care to pregnant women and neonates within the Cape Peninsula. The PMNS consists of primary care facilities known as Midwife Obstetric Units (MOU's), two secondary care facilities namely Mowbray Maternity Hospital (MMH) and New Somerset Hospital (NSH) while Groote Schuur Hospital (GSH) provides tertiary level care. 28000 births occur within the PMNS annually; more than $50 \%$ of the births in this service are managed at MOU's with $33 \%$ of births occurring in secondary facilities and $15 \%$ of the births in the PMNS managed at a tertiary level of care (Van Der Spuy, 2003). The service has protocols for clinical management and care which are shared by all the maternity care facilities within the PMNS, as well as a system of referral between the various levels of care. Referral is facilitated by developed protocols, communication between the various facilities and the availability of transportation to transfer mothers and neonates. This study looks at the response times of ambulances in this setting. The National Committee for the Confidential Enquiries into Maternal Deaths (NCCEMD) recommends that emergency transport be available for all antenatal and peripartum women and their babies who experience complications. The indicator suggested is the time it takes for an ambulance to arrive on the site from where it was summoned within one hour. This recommendation has been the motivation for this study.

\section{Background}

Midwife Obstetric Units are "decentralized obstetric delivery centres" developed by Philpott in the then Salisbury, Rhodesia now called Harare, Zimbabwe (Gunston \& De Groot, 1975: 1147). At this level of care uncomplicated pregnancies and births are managed. At the core of the functioning of the MOU is the registered midwife who performs the initial risk assessment and only keeps "low risk" patients for antenatal, intrapartum and postnatal care at the facility (Van Coeverden de Groot, 1993: 225). Midwives at MOUs are expected to be "competent in detecting abnormalities and providing emergency care", if necessary, before referring high-risk patients to the next level of care. The MOU also functions as a first "port of call" in an emergency (Van
Coeverden de Groot, 1993: 225) if and when experienced by pregnant women in the community. MOUs have no resident medical staff (Van Coeverden de Groot, 1993: 225) and midwives at these facilities depend on the Emergency Medical Services (EMS) to transport high risk patients and emergencies needing higher levels of care.

There are presently six MOU's in the PMNS providing antenatal, postnatal and 24 hour labour care to low risk mothers and neonates. Three of these run satellite clinics for antenatal and postnatal care. Staff from the MOUs service these clinics for specific sessions during the week. Since this study was conducted, one satellite facility has been closed due to staff shortages. All the facilities have specified referral centres to which they refer high-risk patients, complications and emergencies. Figure 1 shows the referral system structure.

The guidelines for the referral of patients include a system of prioritizing calls for the transfer of women in the peripartum period. At the time that this study was conducted, the most recent document explaining the various priorities of calls was departmental notice $27 / 1987$ of the University of Cape Town Department of Obstetrics and Gynaecology.

\section{Figure 1: PMNS referral structure}

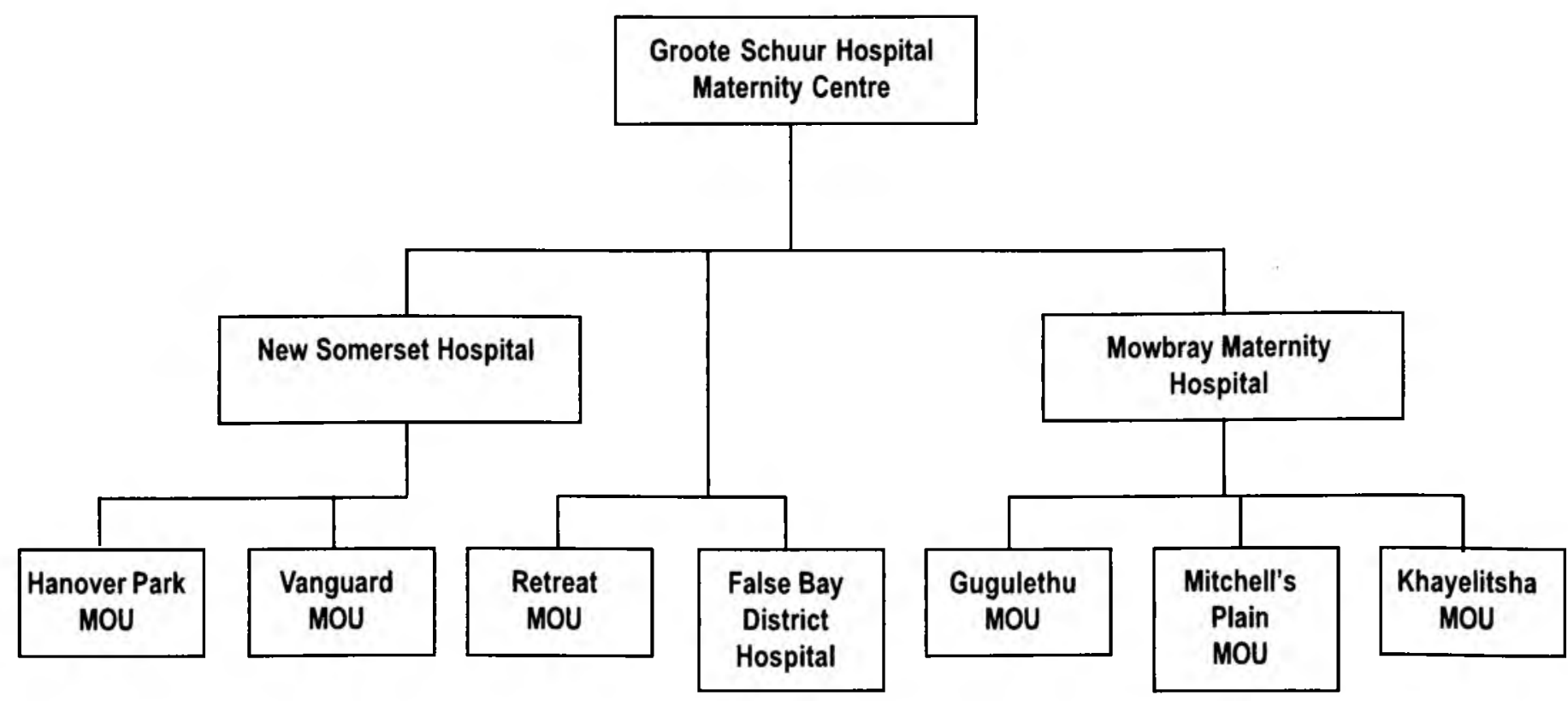


The various priorities were:

"Ordinary/Routine transfer: e.g. prolonged latent phase of labour, nonproteinuric hypertension, preterm prelabour rupture of membranes.

Urgent transfer: e.g. preterm labour with $<5 \mathrm{~cm}$ dilated, retained placenta with no active blceding.

Flying squad: e.g. imminent eclampsia, eclampsia, suspected abruptio, prolapsed cord.

Neonatal: e.g. neonatal respiratory distress."

No target times for response times were specified in this document. The types of obstetric and foetal emergencies which constitute flying squad calls are managed by midwives in MOUs before referral and emergency services personnel en route to referral hospitals.

The second 'Saving Mothers' report on Confidential Enquiries into Maternal Deaths in South Africa has recommended that emergency transport facilities must be available for all pregnant women with complications at all sites (Department of Health, 2002). The indicator to be used to assess the fulfillment of this recommendation is the time taken from the placement of the call to the arrival of the ambulance at the facility from where the patient is to be collected. The target to have been reached by December 2004 was $50 \%$ of ambulances to arrive at the facility within one hour of the call being made (Department of Health, 2002). The motivation for this is that transport delays were directly related to a significant proportion of maternal deaths. This report further asserts that transport problems between facilities contributed to $6.6 \%$ percent of the assessable maternal deaths in the Western Cape during the triennium 1999-2001 (Department of Health, 2002). This figure was one of the lowest, after the Free State, compared to other provinces for this particular avoidable factor. The Cape Peninsula is particularly well provisioned in terms of infrastructure such as roads and communication as well as the existence of the PMNS system and protocols.

Responsibility for the provision of emergency medical services through- out the Western Cape lies with the provincial government (Veriava \& Curnow, 1997: 6). The Cape Peninsula forms the most south-western part of the Western Cape. Media reports have suggested that delays are being experienced in the response of ambulances to calls (Smetherham, 2004). This report alluded to the delays being a result of dire budgetary constraints being experienced by the EMS. It has been asserted that the restructuring in the health system has necessitated patients being transferred from one facility to another and that $40 \%$ of ambulances spend their time undertaking interhospital transfers (Veriava \& Curnow, 1997:7). The assumption made here is that not all interhospital transfers constitute emergencies requiring an ambulance. The use of the emergency medical services for the transfer of patients being referred for tubal ligation also needs to be reconsidered.

The migration of people seeking health care in the province has significant cost and administrative implications for the health department in the Western Cape (Human, Kroon, Bergman, Fawcus \& Ntwana 2003: 634). This means that there are increased numbers of people, not necessarily permanently resident in the Western Cape, who rely on and therefore add to the workload of the EMS. Mongwe asserts that people are seeking help in Western Cape Health facilities, because of the perception that a "higher level of service" exists when compared with the Eastern Cape (2002).This adds to the load that needs to be carried by health care services in the Western Cape including the emergency medical services. In addition violence is being directed towards EMS personnel in certain areas (Kassiem, 2005). This means that staff subjected to acts of violence in the course of their duties, may have to be removed from response duties in order to deal with these violent acts directed at them. This influences the number of personnel available to staff vehicles adding to the workload of an over-burdened service.

Anecdotal reports have shown a wide range of response times by ambulances to calls made from MOUs to transport patients as referrals to other facilities providing higher levels of care. Response times have been acknowledged by Dr. Cleeve Robertson to be a "criti- cal issue" (Smetherham, 2004).

\section{Problem Statement}

Response times of ambulances to calls from MOUs, although varied, are perceived as slow. Delays in transporting women experiencing complications during their pregnancies, births or in the postnatal period to higher levels of care may have negative consequences such as fetal, nconatal or maternal morbidity or death.

\section{Aim of the study}

The intention of the study was to determine the response times of ambulances to maternity and neonatal specific calls from MOUs within the PMNS.

\section{Study Objectives}

1. To establish the response times of ambulances to calls from MOUs in the PMNS for all (Obstetric and Neonatal) transfers.

2. To compare the ambulance response times to various MOUs.

3. To establish the response times of the various priorities of calls i.e. ordinary ambulance, urgent ambulances and "flying squad".

4. To compare ambulance response times with the target set by the 'Saving Mothers' report of the National Department of Health.

\section{Literature review}

Searches using PubMed(B) and Biblioline ${ }^{\circledR}$ were made to determine what previous work has been done on the response times of ambulances to maternity care facilities and on ambulance responses to domestic calls and the pre-hospital environment. The search terms used were: "PMNS", "ambulance response times", "maternal transfer" "Cape Town", "obstetric emergency" and "peninsula maternal and neonatal service" Many of these studies looked at the implications of quick responses on mortality and morbidity in settings other than maternity care. The only published work that could be found relating to the responses of ambulances to interhospital transfers in the maternity setting, was by Van Coeverden De Groot, Van Coeverden De Groot, Smith and Isaacs in 1994 who reported that ambulance 
delay was "disturbingly common" in transferring patients from MOUs with poor progress in labour. They asserted that ambulance delays are the most significant contributors to the delay experienced in the transfer of patients from MOUs to hospitals. The findings relate to the length of time between "summoning the ambulance to the MOU and the patient's first assessment at the hospital". These delays were compared to hospital procedural delays (Van Coeverden De Groot et al, 1994:73). The study did not assess the length of time it took from the first call to request an ambulance to the arrival of the ambulance at the facility.

Gibson, Bailey and Ferguson (2001:300) assert that transporting mothers with fetuses in-utero to higher levels of care can be regarded as a key strategy in improving the survival of high-risk infants. It has also been argued that the use of guidelines for the transfer/referral of pregnant women improves the outcome for babies in terms of morbidity and mortality (Gibson et al, 2001:300). The use of protocols is a key feature of the PMNS.

\section{Methodology}

An exploratory descriptive study was undertaken to investigate the response times of ambulances to MOUs within the PMNS. This study design "provides the least control over variables" (Brink \& Wood, 1998: 283) because "the situation, not the investigator, controls the data" (Brink \& Wood, 1998: 285). In this case the variable was the response times of ambulances to calls from the MOUs. The absence of knowledge about the behaviour of the variable did not allow for the use of a theoretical base for the study thus making this study design appropriate for this investigation.

Ethical approval to conduct the study was granted by the Faculty of Health Sciences Research Ethics Committee of the University of Cape Town.

The study was conducted in five MOUs in the PMNS which are all based in the Cape Town metropolitan are. All these MOU's are under the administrative control of Mowbray Maternity Hospital in Cape Town. Permission to conduct the study was granted by the medical and nursing administration of Mowbray Maternity Hospital. Two units were excluded from the study-one because it does not have a labour ward (providing only antenatal and post natal care) and the other because medical staff on the premises could assist midwives in the event of an emergency although this facility provides primary maternity care.

\section{Study sample}

The study sample consisted of 10 response times of ambulances being recorded to each of the 5 MOU's included in the study. This sample size was calculated using Stata ${ }^{\text {TM }}$ software. The projected average response time was anticipated to be 60 minutes, based on the NCCEMD guidelines, with a standard deviation of 20 minutes. The minimum sample size was 43 recordings for all the MOUs included in the study which would give a statistical power of $90 \% .10$ consecutive response times were recorded in each of the 5 MOUs, giving a total sample size of 50 responses.

\section{Instrument}

Data was collected by means of a tabulated $\log$ sheet. The instrument required the recording of the time a call was placed to the ambulance control room to transfer a patient, the reason or diagnosis, the priority of the call in terms of whether an ordinary, urgent or flying squad was requested and the time of the arrival of the ambulance. Space was allocated to record the last three/four digits of the patient folder number.

\section{Validity}

The measurement of the time it takes for ambulances to respond to calls placed from the MOUs on the instrument ensured face validity. Clocks are valid instruments for measuring time. Each MOU had available, working clock/s. The instrument therefore measured what it was supposed to measure (response times of ambulances) which is a criterion for face validity (Brink, 2002: 168).

\section{Reliability}

All data was gathered during the same time frame with all units commencing data collection during the same week.
All facilities had functional labour ward clocks which were used to note the times to ensure the reliability of the data. On the day of commencing data collection, the various facilities were visited to ensure that all clocks were in working order, as was confirmed the week prior to data collection. The recorded data could be verified against the labour ward registers in each facility thus adding to the reliability of the data, this was facilitated by using the last three/four digits of the patient record number to check against the labour ward register and to ensure that one entry per patient was made. Facilities using the "Cradle" patient information management system were advised to use the last four digits of the patient record.

\section{Pilot study}

A pilot study was conducted at one MOU to assess the feasibility of the research project and user-friendliness of the data instrument. This MOU, although under a different management structure, functions under the same clinical policies and protocols as the other MOUs included in the study. This facility is also dependant on the Metro emergency services to transfer patients to higher levels of care, thus allowing a study of the same variable of response times on the same population of ambulances. Permission was sought and granted from the institutional heads to conduct the pilot study at the facility. The unit manager was approached to explain what was required and the staff briefed about what was needed to be recorded. Data was to be collected on both day and night shifts. An information leaflet, detailing what was required and the researcher's contact details, was left at the facility. The briefing visit brought to light the absence of wallmounted clocks in the facility. Four clocks with batteries were purchased by the researcher and handed to the unit manager for mounting in the labour ward to ensure accurate time-keeping.

No problems were reported with the use of the instrument as a recording mechanism. What came to light during the pilot study was that on one occasion an ambulance arrived to transfer a patient, but then was unable to take the patient because the condition of the 
patient did not allow the EMS crew to "load" the patient as other, non-obstetric patients in the ambulance were needing to be transferred to facilities other than the referral hospital of the unit. The implication of this situation was that waiting time was increased because another vehicle would need to be dispatched to transfer the patient. The decision was made to include these situations when recording the data and to note the time of the first call and the time of arrival of the ambulance that takes the patient to the referral hospital. During the pilot study a further exclusion criterion was identified; patients requiring transfer for tubal ligation were excluded from the sample as these did not require definitive intervention influencing morbidity and mortality. Data collection for the pilot study took three days to complete after which the completed instrument was collected and minor changes made.

\section{Ethical and legal considerations}

No patient identifying data was collected thereby conforming to the ethical principle of confidentiality. No significant time commitment was required from already overworked MOU staff in order to record the data to detract from patient care and other duties. The data collection period lasted less than one week and was therefore not significantly inconvenient to staff. No risk to patients could be identified in the data collection process. A potential benefit to patients is that this study could inform health service policy makers and leaders around improving emergency transport capacity in the Cape Peninsula. A potential benefit for the service is that this study provides evidence of one of the problems experienced by the staff in primary maternity care.

\section{Data collection}

Coordinators managing the various MOUs were briefed by the researcher on the data collection procedure and the data collection instrument was distributed at this briefing. This information was then relayed by the facility coordinators to the facility managers, who were then contacted telephonically by the researcher to reinforce the data collection procedure. Data collection was commenced simul- taneously in September 2005 and this was ensured by a telephonic reminder by the researcher to all the units included in the study. The availability of functioning clocks in the labour wards was confirmed with the respective facility managers prior to the commencement of the study.

On the day of the commencement of data collection, facilities were visited in the afternoon to follow up any problems or queries that may have existed. None were reported. The follow up visit also served as an opportunity to check that the clocks in all the facilities were in working order to ensure reliability of the data.

The data collection period ranged from two to five days at the various MOUs. There was no data collection over a weekend or during a week with a public holiday as this was specifically excluded when choosing a week for the sample; these periods were assumed to place extra demands on the emergency medical services due to increases in alcohol-related motor vehicle accidents and violence. Completed data collection instruments were then collected after being notified by the facility managers that they were complete. The collected data could then be verified by correlating the information with the facility admission register.

\section{Data analysis}

The collected data was entered into an Excel® spreadsheet and the response times in minutes were calculated. Two data entries from two different units were excluded from the data analysis because one patient with a diagnosis of preterm labour had given birth at the facility prior to the arrival of the ambulance which was summoned $115 \mathrm{~min}$ utes earlier. The other excluded entry was for a call to transfer a patient with preterm rupture of membranes, whose family decided to transport her themselves to the referral hospital $120 \mathrm{~min}$ utes after the ambulance was called. The sample size was therefore 48 calls, which met the required minimum of 43 responses. Mean, median and standard deviation of the response times per unit as well as the whole sample of 48 calls were determined.

The priority categories of calls were analysed in the same way. The median was used in comparing the response times to the various categories of calls and various MOUs because the distribution was uneven, thereby giving a more accurate picture of the data as opposed to using the mean (Brink, 2002: 185). The Kruskal-Wallis test was applied to determine the P-value to ascertain whether there were any statistically significant differences in the median response times of ambulances to the various units and various categories of calls. A P-value of $<0.05$ would indicate a statistically significant result.

\section{Results}

The response times of all the calls from the five MOUs included in the study are presented in table 1 . The response times for all calls $(n=48)$ had a mean of

\section{Table 1: Overall response times in minutes for all MOUs}

\begin{tabular}{|l|l|}
\hline Mean & 106.67 \\
\hline Median & 82.50 \\
\hline S.D. & 77.28 \\
\hline Range (min-max) & $10-330$ \\
\hline & \\
\hline & $=48$
\end{tabular}

106.67 minutes with a median of 82.5 minutes. The range was 320 minutes.

The response times to individual units are presented in table 2 . The median response times to the various units range from 64 minutes to 120 minutes. A P-value of 0.8940 was calculated. Indicating no statistically significant difference in the median response times to the various units, therefore no MOU is disadvantaged in relation to the others in terms of response times.

The response times to the various priorities of calls is presented in table 3 . The shortest median response time was for urgent ambulances (median= $65 \mathrm{~min}$ utes); the longest median response time was for neonatal calls with a median of 185 minutes. The Kruskal-Wallis test was also applied to determine if there was any statistical difference between 
Table 2: Comparison of response times in minutes between units

\begin{tabular}{|l|l|l|l|l|l|}
\hline \multirow{2}{*}{} & $\underline{\text { MOU1 }}$ & $\underline{\text { MOU2 }}$ & $\underline{\text { MOU3 }}$ & $\underline{\text { MOU4 }}$ & $\underline{\text { MOU5 }}$ \\
\cline { 2 - 6 } & $\mathrm{n}=10$ & $\mathrm{n}=9$ & $\mathrm{n}=10$ & $\mathrm{n}=9$ & $\mathrm{n}=10$ \\
\hline Mean & 93.5 & 100 & 123 & 110 & 106.5 \\
\hline Median & 64 & 65 & 120 & 85 & 85 \\
\hline $\mathrm{SD}$ & 73.85 & 93 & 75.58 & 81.55 & 89.29 \\
\hline Range (min; $\max )$ & $15-220$ & $35-275$ & $20-240$ & $10-240$ & $10-330$ \\
\hline
\end{tabular}

$\mathrm{P}=0.8940$

Table 3: Comparison of various priorities of calls

\begin{tabular}{|l|l|l|l|l|}
\hline & O/A & U/A & E/S & Neo \\
\cline { 2 - 5 } & $\mathrm{n}=17$ & $\mathrm{n}=12$ & $\mathrm{n}=14$ & $\mathrm{~N}=5$ \\
\hline Mean & 95.65 & 115.67 & 87.93 & 175 \\
\hline Median & 85 & 65 & 75 & 185 \\
\hline SD & 73.91 & 91.44 & 61.93 & 70.98 \\
\hline Range (min; max) & $10-330$ & $20-275$ & $10-220$ & $75-240$ \\
\hline
\end{tabular}

$P=0.1893$

$\mathrm{O} / \mathrm{A}=$ ordinary ambulance, $\mathrm{U} / \mathrm{A}=$ urgent ambulance, $\mathrm{F} / \mathrm{S}$ flying squad, $\mathrm{Neo}=$ neonatal

Table 4: Comparison of data with 2002 "Saving Mothers" report target

\begin{tabular}{|l|l|l|l|}
\hline Category of call & $\mathbf{\%}<\mathbf{6 0} \mathbf{~ m i n}$ & $\mathbf{\%}>\mathbf{6 0} \mathbf{~ m i n}$ & No. $<\mathbf{6 0} \mathbf{~ m i n}$ \\
\hline Ordinary ambulance & $41 \%$ & $59 \%$ & 7 out of 17 calls \\
\hline Urgent ambulance & $42 \%$ & $58 \%$ & 5 out of 12 calls \\
\hline Flying squad & $43 \%$ & $57 \%$ & 6 out of 14 calls \\
\hline Neonatal & $0 \%$ & $100 \%$ & 0 out of 5 calls \\
\hline All calls & $35.5 \%$ & $64.50 \%$ & 18 out of 48 calls \\
\hline
\end{tabular}

the median response times of various categories of calls. A P-value of 0.1893 was calculated, indicating no statistically significant difference in the response times of the various priorities of calls.

Comparing the data to the recommendations and target set by the 2002 'Saving Mothers' report is presented in table 4 . The recommendation was that by
December $2004,50 \%$ of ambulances summoned should arrive at the facility within 60 minutes. In this sample $35.5 \%$ of the ambulances arrived within 60 minutes. If the neonatal calls are excluded from the calculation then $41.8 \%$ of the ambulances arrived within 60 minutes in the other categories of calls.

\section{Discussion}

This study was planned and conducted under optimum conditions where there was no public holidays or weekends. Such conditions would place extra demands on the emergency medical services with increases in trauma related emergencies i.e. these results illustrate an optimum scenario. It would mean that during such periods the response times to calls from MOUs could be assumed to increase due to the extra demands on the EMS. The absence of statistical differences between the response times of the various priorities of calls is cause for concern, because it can be inferred that there is no meaningful benefit to the way calls are prioritised in the present system. Flying squad calls, which are requested for the transfer of severely compromised mothers and foetuses, appear to carry no advantage in the transfer of patients needing definitive urgent care.

The appropriate communication between control room and responding ambulances was brought into question during the pilot study, when an ambulance that was "too full" to take a patient arrived at the MOU. This situation prolongs the waiting time to transfer patients, as another ambulance needs to be despatched to ultimately transfer the patient.

None of the neonatal calls included in the sample $(n=5)$ had a response time of less than 60 minutes. Prior to the commencement of this study a dedicated neonatal transfer ambulance has been established which has an incubator, neonatal monitoring equipment and paramedic "on board". It has been elicited that this vehicle has wide geographical area of responsibility including Atlantis and Wellington. This vehicle is also sometimes diverted to respond to non-neonatal calls if a vehicle or paramedic is required (Personal communication, 23/10/2005) ${ }^{1}$. This presents an area of concern and could contribute to the delayed response times in this sample, although small, of neonatal calls. There is no published recommendation for the response time for this category of call in either the "Saving Mothers" or "Saving Babies" reports.

National norms for the response times of ambulances, as contained in the METRO EMS annual review of 2003, suggest a response time of 15 minutes 
in "built-up areas" as ideal. This study proves that response times to MOUs in the PMNS fall short of this "norm". The implications of violence directed at EMS personnel, as reported in the media, are that if certain areas are declared 'no-go' areas (Karriem, 2005), it may further compromise the transfer of mothers and babies from MOUs in these areas.

The 1987 departmental notice of the University of Cape Town department of Obstetrics and Gynaecology states that flying squad transfers require the escort of a registrar or house officer. This however is not the case in the present staffing climate of the PMNS as the despatch of a medical officer in such cases was discontinued some years ago.

The recommendations of the NCCEMD provide a more 'lenient' time frame of $50 \%$ of ambulances to arrive within one hour. This study shows that this recommendation is also fallen short of in the PMNS. More so now since the recommendations and target have been revised in 2006 for $70 \%$ of ambulances to arrive within 60 minutes of a call.

\section{Limitations of study}

Although the study sample was small, it was calculated on the basis of $90 \%$ power and was conducted over a short period of time under what was considered to be 'ideal' circumstances where no extra demands are made on the EMS in terms of weekends and public holidays.

There was dependence on the staff working in the MOUs for the collection of data which is considered a procedural limitation. The reliability of the data from one facility was questioned because there were no data recordings on the instrument for a period of 18 hours despite there having been transfers (two for the period) noted in the labour ward admission register. The recorded data on the instrument was however used in the analysis. The 'missed' entries were not considered to be significantly influential on the findings because although two consecutive calls were omitted the reasons for the calls and time frame of responses did not appear very different to those recorded on the data instrument.

Flying squad calls have an added time lag because these calls are placed via the Groote Schuur labour ward by the senior obstetric registrar "on-call" and not directly from the MOU clinician. Somerset and Mowbray Maternity Hospitals also place flying squad calls from their respective MOUs through this route thereby adding time to the response time for this category of call if not done immediately. This may have influenced the recorded response times for this category of call because up to three people would be involved in making three calls to transfer a single patient. This chain of calls may influence the ultimate time it takes an ambulance to respond to this category of call. The study did not attempt to measure this pattern and its influence on the findings.

This study did not look at the causes of quick or delayed responses, neither did it report on the clinical outcome of the patients who were transferred, as these were beyond the scope of this study.

\section{Recommendations}

The system of call prioritisation in the PMNS and EMS at the time that this study was conducted needs revision. The system of prioritisation of the various categories of calls does not appear to be the same in the PMNS and EMS.

Call prioritisation in the EMS was previously "allocated by the caller" (Dr Wayne Smith, 2005, personal communication). The need for having flying squad calls placed via the Groote Schuur Hospital labour ward added to the time delay when needing to have patients transferred for urgent, higher levels of care. This should be reassessed when revising the policy, so that the placing calls for this category may be undertaken by the medical staff of the secondary referral centres
(Mowbray Maternity and Somerset Hospitals). The present list of diagnoses that constitute 'flying squad' emergencies can be used as a guide by the staff at secondary maternity facilities. Since this study was conducted the call prioritisation criteria have been amended and were introduced in the EMS and PMNS in April 2006. No link could however be established between the findings of this study and the revision of the transfer protocols.

If delays are due to capacity or resource constraints, community mobilisation and public/private partnerships could be considered. The communities served by the MOUs all have established transport infrastructures such as roads. The feasibility and availability of using community members or private contractors to transport non-emergencies to higher levels of care may be worthwhile investigating. Diagnoses such as prolonged pre-labour rupture of membranes and neonatal jaundice may be considered to be transported in this way. This situation could indicate that this is not an appropriate use of the EMS, where private contractors could be used to transfer patients not requiring medical monitoring en route. This may relieve the patient load on the EMS and allow more effective use of material and human resources. In some cases it may be feasible and appropriate to use the families of patients who have vehicles for the transfer of stable patients to higher levels of care. These kinds of transfers can be considered on an individual basis depending on the severity of the problem and discussion between relevant referring and accepting clinicians.

In order to improve the situation as illustrated in the findings of this study, public-private partnerships with private sector emergency services should be investigated. The circumstances under which a private ambulance could be summoned e.g. in terms of time delay should be determined. This should be done after careful evaluation of the cost implications for the provincial health department in these situations.

\footnotetext{
'Information elicited from EMS staff member on condition of anonymity.
} 
It may be of value to conduct a larger scale study over a longer period of time and to investigate the implications and consequences of delayed or quick response times on maternal and neonatal morbidity and mortality in the PMNS. It may also be worthwhile to conduct a comparison with other regions and provinces, providing an opportunity for further, more extensive research on this topic.

\section{Conclusion}

Ambulance response times in the PMNS are cause for concern as proven in this small but significant study. It confirms the perception of delays experienced in the MOUs of the PMNS. The Western Cape is considered to be better resourced than other provinces and yet the time it takes to transfer maternal and neonatal emergencies leaves considerable room for improvement.

\section{References}

BIGELOW, LK; ANDERSON, KC \& CAMP, J 1997: Perinatal Transport Casebook, Journal of Perinatology. Vol. 17, No. 5: p411-415.

BRINK, HI 2002: Fundamentals of Research Methodology for Health Care Professionals, Juta \& Co, Lansdowne.

BRINK, PJ; WOOD, MJ 1998: Advanced Design in Nursing Research, $2^{\text {nd }}$ edition, Sage Publications, California.

MEDICAL RESEARCH COUNCIL (SOUTH AFRICA) 2000: Saving Babies: A Perinatal Care Survey of South Africa, Pretoria.

GIBSON, M; BAILEY, C \& FERGUSON, $J$ 2001: Transporting the incubator: Effects upon a region of the adoption of guidelines for high-risk maternal transport, Journal of Perinatology, 21:p300-306

GUNSTON, K \& VAN COEVERDEN DE GROOT, HA 1975: The Cape Town Obstetric Flying Squad, South African Medical Journal. Vol. 49: 1147-1148.

HUMAN, I; KROON, M; BERGMAN, N; FAWCUS, S \& NTWANA, B 2003: Patient population movement in a Cape Town obstetric service, South African
Medical Journal, 93(9): 634.

KARRIEM, A 2005: Police may soon escort ambulances after attacks. Cape Times: 24 October.

MONGWE, R 2002: Migration Study in the Western Cape: Residents' perceptions regarding migration and social service delivery (especially health and education): Case studies in George and Cape Town. Available online at www.capegateway.gov.za/Text/2003/ migration study 20017 education+ health.pdf accessed 12/09/2005.

PROVINCIAL GOVERNMENT WESTERN CAPE 2003: Medical Emergency Transport and Rescue Organisation (Metro EMS) Annual Review 2003. Available online at: http:// www.capegateway.gov.za/Text/2004/ 11/ar ems 2003.pdf. Accessed 05/07/ 2004.

PROVINCIAL GOVERNMENT WESTER CAPE 2003: Community Health Services Organisation Annual Report April 2002-March 2003. Available online at: $\underline{\mathrm{http}: / /}$ www.capegatewav.gov.za/Text/2004/3/ chso annual report 2002 3.pdf Accessed 18/06/2004.

SOUTHAFRICA(REPUBLIC) 2006: Ten Key Recommendations, available online at http://www.info.gov.za/ otherdocs $/ 2006 /$ maternal deaths.pdf accessed 16/01/2007

SOUTHAFRICA(REPUBLIC) 2002: Saving Mothers: Second Report on Confidential Enquiries into Maternal Deaths in South Africa: 1999-2001. Government Printer: Pretoria

SOUTH AFRICA (REPUBLIC) 2002: Guidelines for Maternity Care in South Africa: A Manual for Clinics, Community Health Centers and District Hospitals, $2^{\text {nd }}$ edition, Government Printer, Pretoria.

SMETHERHAM, J 2004: Deaths daily as ambulance service battles to keep pace. Cape Times, 16 September.

UNIVERSITY OF CAPE TOWN 2004: Division of Obstetrics and Gynaecology, http://web.uct.ac.za/depts/dri/ resrep01/faculties/health/obs/ obs01.html Accessed 18/06/2004.
VAN COEVERDEN DE GROOT,AA; VANCOEVERDEN DE GROOT, HA; SMITH, CM \& ISAACS, S 1994: Referrals for inadequate progress of labour from Midwife Obstetric Units to the referral hospitals in Cape Town, Curationis, 17(4): 71-74.

\section{VAN COEVERDEN DE GROOT, HA} 1993: Provision of a Community Perinatal Service in a Developing Country, The Australian \& New Zealand Journal of Obstetrics and Gynaecology, 33 (3): 225-229.

VAN DER SPUY, Z 2003: Division of Obstetrics and Gynaecology, online from www.gsh.co.za/pc/obs.html accessed 18/06/2004.

VERIAVA, F, CURNOW, P 1997: Special Report: An Investigation into the Prevailing Conditions at Ambulance \& Rescue Services. Online from w w w.s a h r c.org. $\mathrm{z}$ a / section 27 case 1.pdf accessed 10/ $11 / 2004$. 